\title{
Analysis of Clinical Features of Ocular Presentation in Cranial Venous Sinus Thrombosis
}

\author{
D. Wang ${ }^{-1}$, B. Fang ${ }^{2}$, S. Wei ${ }^{1}$ \\ ${ }^{1}$ Department of Ophthalmology, Chinese PLA General Hospital, Beijing 100853, China \\ ${ }^{2}$ Department of Neurology, First Affiliated Hospital of Liaoning Medical College, Jinzhou 121001, China
}

\begin{abstract}
Background: To recognize ocular presentations in cranial venous sinus thrombosis (CVST) which were easy to be misdiagnosis.

Design: Retrospective study.

Methods: Review clinical informations including general informations, general performances, and ocular presentations of 118 inpatients with CVST in the general hospital of chinese people's liberation army during 2005-2009.

Main Outcome Measures: The ocular symptoms as the initial onset presentations or simultaneous phenomenon among different onset type patients were analyzed.

Results: Of all the CVST patients, 21.2\% (25/118) presented with ocular symptom as the initial presentation, 30.5\% (36/118) presented with ocular symptom as well as the other symptoms, and 48.3\% (57/118) presented with non-ocular symptoms as the initial onset. The CVST patients were divided into 3 groups according to the onset type. There was no marked statistical significance among groups. The most common major complaints were blurring and degeneration of acute vision, accounting for $85.9 \%(61 / 71)$ of all abnormal ocular chief complaints. The most common objective sign in eyes was papilloedema, accounting for $48.3 \%(57 / 118)$ in this group of CVST patients. About 22.4\% (13/58) showed acute vision deterioration at 1-year follow-up, due to optic atrophy.

Conclusions: As ophthalmologists, we should master the onset characteristics and clinical manifestations of CVST. Early diagnosis and treatment is very important for the prevention of vision deterioration, especially for patients with ocular syndrome as the initial onset syndrome. For isolated agnogenic intracranial hypertension, we should consider the possibility of CVST.

Keywords: Cranial venous sinus thrombosis; Ocular presentations; Papilloedema
\end{abstract}

\section{INTRODUCTION}

Cranial venous sinus thrombosis (CVST) is a specific type of cerebrovascular disease. Patients present signs of intracranial hypertension, such as headache, vomiting, and papilloedema, which are common clinical manifestations. Because ocular onset presentation is the most common symptom, some patients may be misdiagnosed or a missed diagnosis may occur if only ocular onset presentation occurs as the initial onset symptom. In recent years, the popularity of some technical applications, including magnetic resonance imaging (MRI), magnetic resonance venography (MRV), and digital subtraction angiography (DSA) has resulted in ongoing improvements in diagnosis and increased levels of awareness of this disease. Although there are many reported clinical observations of CVST [1-4], there are few reports on the study of the interrelationship between the clinical manifestations and ocular onset presentation. To this end, we collected the information of 118 inpatients with CVST and treated the data with a retrospective analysis for the period 2005-2009. We expected that it would provide more clinically significant data for the early diagnosis and treatment of CVST.

\section{Methods}

The aim was to study 118 inpatients with a retrospective analysis, in the Department of Neurology and Interventional Section, The General Hospital of Chinese People's Liberation Army, during 2005-2009. Of 118 CVST patients, the numbers of men and women are 53 and 65 , respectively; the gender constituent ratio is $1: 1.2$ (men: women). The onset age ranged from 15 to 67 years: average age was $34.9 \pm 10.6($ mean \pm SD) and between 20 and 45 was most common; the initial onset ages were concentrated between 30 and 40 years. Causes of CVST included infectious (the number of cases of upper respiratory tract infection and diarrhea was 12 and the number of cases of otitis media and mastoiditis were 7 and 3, respectively) and non-infectious origins (the number of cases of puerperium, hyperlipidemia, oral contraceptive use, trauma, postoperative or agnogenic were 9, 3, 8, 10, and 66, respectively). Not only the clinical symptoms and systemic examination, but also definitive diagnosis of CVST is mainly identified by DSA (Fig.1). Most of the sites of thrombosis are multiple: $23.7 \%(28 / 118)$ of the CVST patients presented with simple superior sagittal sinus thrombosis; $24.6 \%(29 / 118)$ presented with simple lateral sinus thrombosis; $3.4 \%$ (4/118) presented with purely straight sinus thrombosis; $26.3 \%(31 / 118)$ presented with superior sagittal sinus thrombosis in combination with lateral sinus thrombosis; $2.5 \%(3 / 118)$ presented with cavernous sinus thrombosis; $3.4 \%$ (4/118) presented with intracranial large in combina- 

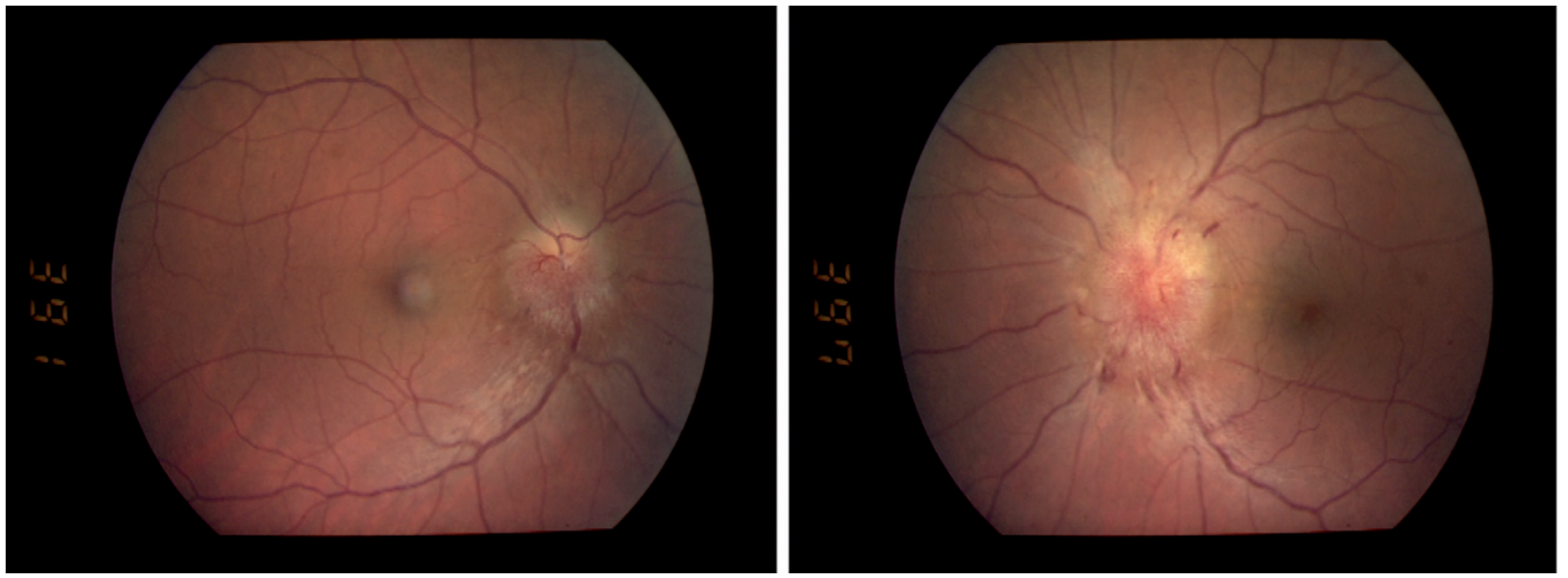

Fig. 1. Photochrome of binoculus with CVST. papilloedema, and varicosity of both eyes. Retinal hemorrhage of the left eye.

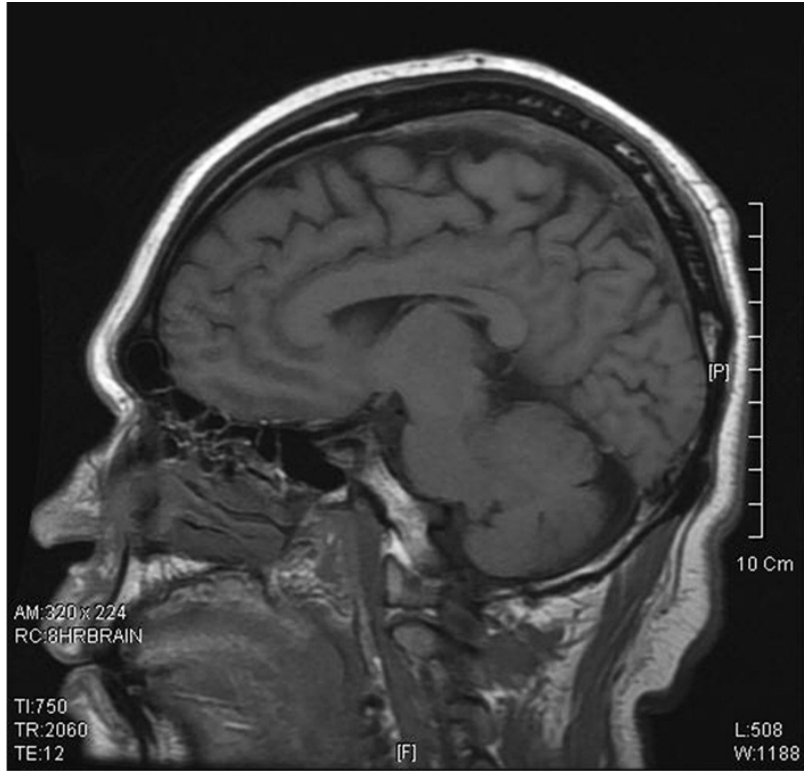

Fig. 2. MRI showing high signals involving the superior sagittal sinus thrombosis on TW1.

tion with small vein thrombosis; $8.5 \%(10 / 118)$ presented with thrombosis in more than 3 sites; and $4.2 \%$ (5/118) of the CVST patients presented not only with thrombosis, but also dural arteriovenous fistula formation. All patients were given a cerebrospinal fluid examination. In these results, the cerebrospinal fluid pressure was measured at up to $700 \mathrm{mmH}_{2} \mathrm{O}$ (1 $\mathrm{mmH}_{2} \mathrm{O}=0.133 \mathrm{kPa}$ ). The results ranged mostly between 300 and $400 \mathrm{mmH}_{2} \mathrm{O}$. Of the 95 patients who received an MRI examination, 42.3\% (41/95) presented abnormal changes, including 10 patients with empty sella (Fig. 2). The eye is mainly given an external ocular examination and fundus examination with a direct ophthalmoscope (Fig. 3). All patients received treatments during hospitalization, such as thrombolytic therapy intervention with transcranial venous sinus intubation, carotid arteriopuncture and anticoagulant therapy with oral anticoagulant. Patients had follow-up inspections for about 1 year. The CVST patients were divided into 3 groups according to the onset style [1,

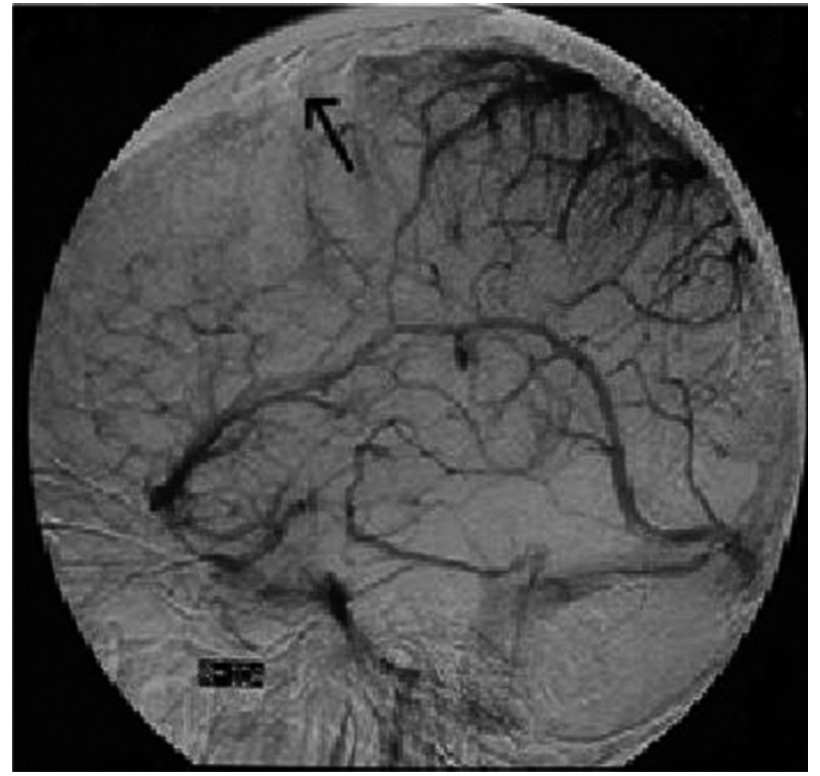

Fig. 3. DSA interal carotid arteriography lateral view did not display the superior sagittal sinus in venous phase (black arrow).

4]. These were acute onset (less than 2 days), subacute onset (between 2 days and 1 month), and chronic onset (more than 1 month). The main components of retrospective analysis were correlation analysis of general information, general performance, and ocular onset presentation of this group of patients, and determining whether there were differences of the ocular symptoms as the initial onset among different onset patients. Statistical analysis was determined using results from SPSS 10.0, using a $\chi^{2}$ test, at a $P<0.05$ level of significance. This study was approved by the institutional review board of the Department of Ophthalmology, General Hospital of Chinese People's Liberation Army (Beijing, China) and written informed consent was obtained from every participant.

\section{RESULTS}

Of the CVST patients, $21.2 \%(25 / 118)$ presented with ocular symptoms as the initial onset. All of these pa- 
Table 1. Initial presentation with different onset style of 118 patients.

\begin{tabular}{lllll}
\hline Onset style & $\begin{array}{l}\text { Ocular } \\
\text { Number }\end{array}$ & $\begin{array}{l}\text { Ocular with other } \\
\text { Number }\end{array}$ & $\begin{array}{l}\text { Other } \\
\text { Number }\end{array}$ & Total \\
\hline Acute onset & 5 & 7 & 11 & 23 \\
Subacute onset & 8 & 11 & 19 & 37 \\
Chronic onset & 12 & 18 & 27 & 58 \\
Total & 25 & 36 & 57 & 118 \\
\hline$\chi^{2}=0.0900$ & & $\gamma=4$ & & $P=0.9990$
\end{tabular}

tients received an ophthalmological examination and CT or MRI as screening tools in order to exclude ocular disease and other intracranial lesions; 30.5\% $(36 / 118)$ of the CVST patients presented with ocular syndrome in conjunction with other syndromes (including headache, dizziness, nausea, vomiting, convulsions, disturbance of consciousness, tinnitus, hearing loss, and limb movement disorder); 48.3\% (57/118) of the CVST patients presented with non-ocular symptoms as the initial onset (mainly headache, nausea, and vomiting). There was no significant difference of ocular presentation incidence as the initial symptom among the 3 groups (Table 1). Of the CVST patients, $60.2 \%(71 / 118)$ presented with ocular onset presentation as the chief complaint. Symptoms included blurred vision, vision loss, transient amaurosis, diplopia, ophthalmalgia, metamorphopsia, and abnormal vision. Of these patients, $85.9 \%(61 / 71)$ presented with blurred vision and vision loss; 30.9\% (22/71) presented with diplopia; 19.7\% (14/71) presented with ophthalmalgia (mainly gas pains). Of the CVST patients, $70.3 \%$ (80/118) presented with an abnormal ocular examination. The CVST patients exhibit not only vision loss, metamorphopsia, and diplopia, but also the symptoms of eyeball movement restriction, conjunctival hyperemia, exophthalmos, nystagmus, and fundus changes. The symptoms of fundus changes include papilloedema, optica trophy, optic disc hemorrhage, retinal hemorrhage, retinal varices, and macular edema. Papilloedema is the most common symptom, account for $68.7 \%(57 / 83)$ of this group of patients. A year later, after the follow-up inspection, there were 58 patients with complete information: $51.7 \%$ (30/58) presented a reduction in ocular onset presentation, and $22.4 \%(13 / 58)$ of these patients presented serious vision loss because of optic atrophy.

\section{DISCUSSION}

DSA with high sensitivity is the key standard of diagnosis of CVST: Accuracy ranges from $75 \%$ to $100 \%$ [5]. Although MRI and MRV have some advantages for checking venous sinus thrombosis, such as being non-invasive, having repeatability and high-resolution, they are not effective with smaller vascular thromboses. Diagnosis using MRI and MRV is difficult also because of the anatomical variants of venous sinus [6]. DSA not only displays the filling state of venous sinus (including symptoms of venous sinus filling defect, underdevelopment, backflow, and anastomosed varicose vein), but also can analyze the cycle time of cerebral arteriovenous cycles in the arterial phase, cap- illary phase, venous phase, and sinus period to confirm which period is delayed when there is an extension of the total cycle time. Because all the patients received the DSA examination to make a definite diagnosis, so the patients we enrolled were accurate and credible. The average age of onset for these patients is $34.9 \pm 10.6$ years, and it affects slightly more females than males-match with the reported literatures [7, 8]. Females aged from 20 to 35 are most commonly affected which may relate to pregnancy, childbirth, and use of oral contraceptive according to other reports [4]. In this study, we do not make any further analysis of the relevant factors of this phenomenon, but there was a study showed that CVST was related to antiphospholipid antibody syndrome [9].

The clinical manifestation of CVST is complex and non-specific. Its main symptoms are intracranial hypertension and cortical damage. Headache is the most common symptom, accounting for $74-90 \%$ of CVST patients; $70-75 \%$ of this group presented with headache as the initial onset symptom $[3,10]$. However, $20 \%-40 \%$ of the CVST patients only presented with benign intracranial hypertension [11]. Over 75\% of patients in this group mainly complained of serious headache, and this corresponds to the reports. It should be noted that $21.2 \%$ of CVST patients presented with ocular syndrome as the initial onset and 30.5\% of the CVST patients presented with ocular syndrome simultaneously with other syndromes in this group. The ocular onset presentation has no relationship to the onset symptoms. So the ophthalmologist must pay attention to, and be familiar with CVST to avoid misdiagnosis and a missed diagnosis when patients present with ocular symptom as the initial onset symptom.

At present, it is believed that underlying ocular symptom of CVST are venous obstruction and intracranial hypertension. The most common ocular symptom is papilloedema. In this group of patients, $48.3 \%$ had papilloedema; this corresponds to the reported $41 \%$ [12]. Unless the macular is affected by retinal hemorrhage, retinal edema, or exudation, there are no identifiable symptoms of early papilloedema, not even vision changes or impaired vision. If the papilloedema caused by intracranial hypertension lasted too long, it would cause progressively deteriorating vision in narrow irregular concentric circles - a result of optic nerve fiber damage. This would eventually result in seriously impaired vision or blindness caused by optic atrophy [13].

The pathogenesis of papilloedema by intracranial hypertension is unclear and controversial. The general- 
ly accepted mechanism is that blockage in axoplasmic flow of the optic nerve causes papilloedema [14]. The prognosis for long-term and serious papilloedema is very poor, so we must actively treat the patient before the papilloedema develops and optica trophy occurs. Once the opportunity for treatment is missed, it would be difficult to retrieve lost vision.

In addition, the intracranial hypertension leads to eighth cranial nerve paralysis and mild cognitive impaired. Some patients presented with diplopia and strabismus because of intracranial hypertension. The cause may due to the eighth cranial nerve having a long itinerary in the cranial cavity, it is easily damaged. This group had 6 patients with diplopia and strabismus. There is a complex relationship between CVST and dural arteriovenous fistula. According to some reports, there may be a dural arteriovenous fistula in the terminal stage of CVST $[15,16]$. In this group, there are 5 patients with dural arteriovenous fistula. These patients were diagnosed with CVST via DSA 6 months to 3 years ago. Each patient presented with the related symptoms of arteriovenous fistula. In addition, the dural arteriovenous fistula may have already existed before thrombosis, so it might be the basic cause of CVST [17].

We have analyzed and summarized the data of 118 patients with CVST over 5 years. This has allowed a deepened recognition of ocular onset presentation of CVST but, in this study, we have not analyzed the relationship between the severity level of ocular onset presentation and the site of thrombosis which need further study.

Conflict of interest: The authors declare that they have no conflict of interest.

\section{REFERENCES}

1 Perez MA, Glaser JS, Schatz NJ. "Idiopathic" intracranial hypertension caused by venous sinus thrombosis associated with contraceptive usage. Optometry. 81(7): 351-8.

2. Wasay M, Kojan S, Dai AI, Bobustuc G, Sheikh Z. Headache in Cerebral Venous Thrombosis: incidence, pattern and location in 200 consecutive patients. J Headache Pain. 11(2): 137-9.

3. Mehndiratta MM, Garg S, Gurnani M. Cerebral venous thrombosis--clinical presentations. J Pak Med Assoc. 2006 56(11): 513-6.

4. Crassard I, Bousser MG. Cerebral venous thrombosis. J Neuroophthalmol. 2004 24(2): 156-63.
5. Nagpal RD. Dural sinus and cerebral venous thrombosis. Neurosurg Rev. 1983 6155-60.

6. White JB, Kaufmann TJ, Kallmes DF. Venous sinus thrombosis: a misdiagnosis using MR angiography. Neurocrit Care. 2008 8(2): 290-2.

7. Ameri A, Bousser MG. Cerebral venous thrombosis. Neurol Clin. 1992 10(1): 87-111.

8. Villringer A, Mehraein S, Einhaupl KM. Pathophysiological aspects of cerebral sinus venous thrombosis (SVT). J Neuroradiol. 1994 21(2): 72-80.

9. Yehudai D, Shoenfeld Y, Toubi E. Looking into the eyes of patients with antiphospholipid syndrome. Clin Rev Allergy Immunol. 2007 32(2): 192-7.

10. Renowden S. Cerebral venous sinus thrombosis. Eur Radiol. 2004 14(2): 215-26.

11. Masuhr F, Mehraein S, Einhaupl K. Cerebral venous and sinus thrombosis. J Neurol. 2004 25111-23.

12. de Bruijn SF, de Haan RJ, Stam J. Clinical features and prognostic factors of cerebral venous sinus thrombosis in a prospective series of 59 patients. For The Cerebral Venous Sinus Thrombosis Study Group. J Neurol Neurosurg Psychiatry. 2001 70(1): 105-8.

13. Rougier MB. [Diagnosing bilateral papilledema]. J Fr Ophtalmol. 33(6): 424-9.

14. Van Stavern GP. Optic disc edema. Semin Neurol. 2007 27(3): 233-43.

15. Malek AM, Higashida RT, Balousek PA, Phatouros CC, Smith WS, Dowd CF, Halbach VV. Endovascular recanalization with balloon angioplasty and stenting of an occluded occipital sinus for treatment of intracranial venous hypertension: technical case report. Neurosurgery. 1999 44(4): 896-901.

16. Phatouros CC, Halbach VV, Dowd CF, Lempert TE, Malek AM, Meyers PM, Higashida RT. Acquired pial arteriovenous fistula following cerebral vein thrombosis. Stroke. 1999 30(11): 2487-90.

17. Fujita A, Nakamura M, Tamaki N. [Multiple dural arteriovenous fistulas involving both the cavernous sinus and the posterior fossa: report of two cases and review of the literature]. No Shinkei Geka. 2001 29(11): 1065-72.

Received: March 23, 2011 / Accepted: May 25, 2011

Address for correspondence:

Dajiang Wang

Department of Ophthalmology

Chinese PLA General Hospital

Beijing 100853

China

Tel.: $\quad+86-13718180877$

Fax: +86-416-4197511

E-mail: wangdajiangv@yahoo.com.cn 